\title{
PT symmetry breaking in multilayers with resonant loss and gain locks light propagation direction
}

Novitsky, Denis V.; Karabchevsky, Alina; Lavrinenko, Andrei V.; Shalin, Alexander S.; Novitsky, Andrey V.

Published in:

Physical Review B

Link to article, DOI:

10.1103/PhysRevB.98.125102

Publication date:

2018

Document Version

Publisher's PDF, also known as Version of record

Link back to DTU Orbit

Citation (APA):

Novitsky, D. V., Karabchevsky, A., Lavrinenko, A. V., Shalin, A. S., \& Novitsky, A. V. (2018). PT symmetry breaking in multilayers with resonant loss and gain locks light propagation direction. Physical Review B, 98(12), [125102]. https://doi.org/10.1103/PhysRevB.98.125102

\section{General rights}

Copyright and moral rights for the publications made accessible in the public portal are retained by the authors and/or other copyright owners and it is a condition of accessing publications that users recognise and abide by the legal requirements associated with these rights.

- Users may download and print one copy of any publication from the public portal for the purpose of private study or research.

- You may not further distribute the material or use it for any profit-making activity or commercial gain

- You may freely distribute the URL identifying the publication in the public portal 


\title{
$\mathcal{P} \mathcal{T}$ symmetry breaking in multilayers with resonant loss and gain locks light propagation direction
}

\author{
Denis V. Novitsky, ${ }^{1,2,3,{ }^{*}}$ Alina Karabchevsky, ${ }^{4,5,6}$ Andrei V. Lavrinenko, ${ }^{7}$ Alexander S. Shalin, ${ }^{2}$ and Andrey V. Novitsky ${ }^{7,8}$ \\ ${ }^{1}$ B. I. Stepanov Institute of Physics, National Academy of Sciences of Belarus, Nezavisimosti Avenue 68, 220072 Minsk, Belarus \\ ${ }^{2}$ ITMO University, Kronversky Prospekt 49, 197101 St. Petersburg, Russia \\ ${ }^{3}$ Saint Petersburg Electrotechnical University “LETI”, 5 Prof. Popova Str., 197376 St. Petersburg, Russia \\ ${ }^{4}$ Electrooptical Engineering Unit, Ben-Gurion University of the Negev, David Ben-Gurion Blvd., P.O.B. 653, Beer-Sheva 8410501, Israel \\ ${ }^{5}$ Ilse Katz Institute for Nanoscale Science \& Technology, Ben-Gurion University of the Negev, Beer-Sheva 8410501, Israel \\ ${ }^{6}$ Center for Quantum Information Science and Technology, Ben-Gurion University of the Negev, Beer-Sheva 8410501, Israel \\ ${ }^{7}$ DTU Fotonik, Technical University of Denmark, Ørsteds Plads 343, DK-2800 Kongens Lyngby, Denmark \\ ${ }^{8}$ Department of Theoretical Physics and Astrophysics, Belarusian State University, Nezavisimosti Avenue 4, 220030 Minsk, Belarus
}

(Received 7 July 2018; published 4 September 2018)

\begin{abstract}
Using the Maxwell-Bloch equations for resonantly absorbing and amplifying media, we study the temporal dynamics of light propagation through the $\mathcal{P} \mathcal{T}$-symmetric structures with alternating loss and gain layers. This approach allows us to precisely describe the response of the structure near the exceptional points of $\mathcal{P} \mathcal{T}$ symmetry breaking phase transition and, in particular, take into account the nonlinear effect of loss and gain saturation in the $\mathcal{P} \mathcal{T}$ symmetry broken state. We reveal that in this latter state the multilayer system possesses a lasinglike behavior, releasing the pumped energy in the form of powerful pulses. We predict locking of pulse direction due to the $\mathcal{P} \mathcal{T}$ symmetry breaking, as well as saturation-induced irreversibility of phase transition and nonreciprocal transmission.
\end{abstract}

DOI: 10.1103/PhysRevB.98.125102

\section{INTRODUCTION}

$\mathcal{P} \mathcal{T}$-symmetric structures and $\mathcal{P} \mathcal{T}$ symmetry breaking seem to be one of the most intensively studied fields in optics and photonics of active systems [1-3]. It dates back to the seminal works of Bender and Boettcher [4,5], who discovered the real-valued spectra of non-Hermitian Hamiltonians in quantum mechanics, provided these Hamiltonians are parity-time $(\mathcal{P} \mathcal{T})$ symmetric, i.e., invariant with respect to simultaneous parity change and time reversal. Such ideas can be straightforwardly transferred to optics using the spatial ordering of passive and active components, thus introducing the concept of an optical $\mathcal{P} \mathcal{T}$-symmetric system.

The simplest optical $\mathcal{P} \mathcal{T}$-symmetric structure can be realized by means of one-dimensional multilayers - similar to photonic crystals - with proper spatial variation of the complex permittivity as $\varepsilon(z)=\varepsilon^{*}(-z)$ (here the asterisk stands for complex conjugation). This condition implies that the real part of the permittivity or refractive index is an even function of the coordinate, whereas the imaginary part is an odd function. Change of the sign of the imaginary part of the permittivity $\operatorname{Im} \varepsilon(z)=-\operatorname{Im} \varepsilon(-z)$ apparently requires amplifying materials. It can be, for instance, an alternation of loss and gain in periodic multilayers. Two principal schemes, longitudinal and transverse ones, are usually employed. In the former system, light propagates directly through the multilayer. In the latter scheme light propagates perpendicularly to the permittivity distribution, along the layer boundaries, being analogous to a system of interconnected waveguides (an optical grating). First theoretical [6,7] and experimental

*dvnovitsky@gmail.com
[8] results on optical $\mathcal{P} \mathcal{T}$ symmetry were reported exactly for the coupled waveguides.

$\mathcal{P} \mathcal{T}$ symmetry allows new ways for controlling radiation fluxes both in optics and plasmonics [9]. A number of effects can be highlighted as a fingerprint of the $\mathcal{P} \mathcal{T}$ symmetry: nonreciprocity of light transmission and beam power oscillations [7], anisotropic transmission resonances [10], unidirectional "invisibility" [11], negative refraction, and focusing of light [12]. $\mathcal{P} \mathcal{T}$ symmetry governs light localization in disordered structures [13]. The usage of gain media raises questions concerning available nonlinear phenomena, such as optical switching and generation of new types of solitons $[14,15]$. Apodization of the refractive index spatial profiles can be used to facilitate switching conditions in $\mathcal{P} \mathcal{T}$-symmetric Bragg gratings [16]. Some nonlinear effects connected to $\mathcal{P} \mathcal{T}$ symmetry have been recently observed in experiments with coherent atomic gases [17]. More prospects are opened by the fact that $\mathcal{P} \mathcal{T}$-symmetric optical gratings are capable of supporting topologically protected bound states [18].

It should be noted that the effects mentioned above may be observable in loss-gain structures lacking the $\mathcal{P} \mathcal{T}$ symmetry. A typical example is the reflectionless transmission and unidirectional "invisibility" for both normal $[19,20]$ and oblique [21] incidence. However, the symmetry breaking at the exceptional points belongs exclusively to the $\mathcal{P} \mathcal{T}$ symmetry domain.

There are a number of phenomena associated with violation of the $\mathcal{P} \mathcal{T}$ symmetry. First, a sharp change in polarization response of the system is possible at the exceptional points [22]. Later an omnipolarizer was designed for converting any light polarization into a given one [23]. Second, enhanced sensitivity of such kinds of systems to external perturbations near exceptional points provides a new approach for sensing 


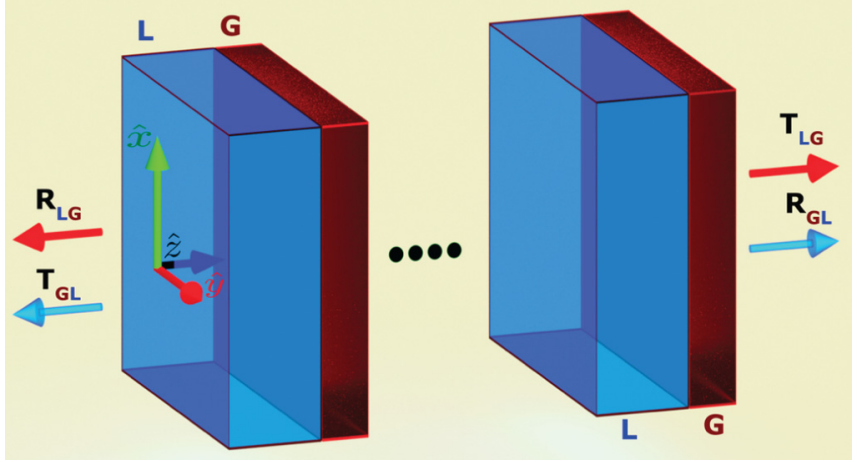

FIG. 1. $N$-periods multilayer system of alternating slabs with loss (L) and gain (G). Transmissions and reflections and corresponding propagation directions are indicated for the waves incident from left-hand ( $T_{L G}$ and $\left.R_{L G}\right)$ and right-hand $\left(T_{G L}\right.$ and $\left.R_{G L}\right)$ sides.

[24,25]. Third, $\mathcal{P} \mathcal{T}$ symmetry breaking plays an important role in laser physics, offering new types of lasers [26-28] and antilasers [29] based on the effect of coherent perfect absorption [30,31]. Finally, the possibility of light stopping at the exceptional point was recently reported [32].

In this paper, we study the phenomenon of $\mathcal{P} \mathcal{T}$ symmetry breaking in one-dimensional multilayers composed of resonantly absorbing and amplifying media. We describe propagation of light in the time domain using the Maxwell-Bloch equations and taking into account loss and gain saturation. The influence of the saturable nonlinearity on nonreciprocity and bistability of $\mathcal{P} \mathcal{T}$-symmetric structures was previously reported both for transverse [33] and longitudinal geometries [34-37]. However, those investigations introduced the saturation phenomenologically via the permittivity. Our approach is based on a self-consistent description of temporal dynamics for both light field and medium loss/gain exhibiting a more realistic treatment of $\mathcal{P} \mathcal{T}$-symmetric optical systems. Therefore, we have a deep insight into peculiarities of the $\mathcal{P} \mathcal{T}$ symmetry breaking and related effects. In particular, we study the lasinglike regime in the $\mathcal{P} \mathcal{T}$ symmetry broken state, where the saturation effects lead to the unique phase transition in the parameter space and nonreciprocal transmission of generated pulses. Pulse-direction locking by the $\mathcal{P} \mathcal{T}$ symmetry breaking in the lasinglike regime is related to the strong light confinement and resembles light polarization locking to its propagation direction in quantum optics [38].

The paper is organized as follows. Section II is devoted to the description of the theoretical model of the loss-gain multilayer and parameters used in calculations. We discuss light behavior in the $\mathcal{P} \mathcal{T}$-symmetric phase in Sec. III by comparing the numerical solution of the Maxwell-Bloch equations and stationary transfer-matrix calculations. In Sec. IV, the $\mathcal{P} \mathcal{T}$ symmetry breaking as a phase transition to the lasinglike regime is studied with emphasis on temporal dynamics of the light propagating through the multilayers. Section V summarizes the article.

\section{RESONANT LOSS AND GAIN MEDIA}

A periodic planar structure composed of $2 \mathrm{~N}$ alternating loss and gain layers shown in Fig. 1 is illuminated by monochromatic light of angular frequency $\omega$ at normal incidence. In this study, both loss and gain are described in the similar manner, using the model of a homogeneously broadened two-level medium. Excluding the rapidly varying factors $\exp (-i \omega t)$ in polarization, population difference, and electric field, we write the Maxwell-Bloch equations [39] for slowly varying amplitudes of these quantities as

$$
\begin{gathered}
\frac{d \rho}{d \tau}=i l \Omega w+i \rho \delta-\gamma_{2} \rho, \\
\frac{d w}{d \tau}=2 i\left(l^{*} \Omega^{*} \rho-\rho^{*} l \Omega\right)-\gamma_{1}\left(w-w_{e q}\right), \\
\frac{\partial^{2} \Omega}{\partial \xi^{2}}-n_{d}^{2} \frac{\partial^{2} \Omega}{\partial \tau^{2}}+2 i \frac{\partial \Omega}{\partial \xi}+2 i n_{d}^{2} \frac{\partial \Omega}{\partial \tau}+\left(n_{d}^{2}-1\right) \Omega \\
=3 \alpha l\left(\frac{\partial^{2} \rho}{\partial \tau^{2}}-2 i \frac{\partial \rho}{\partial \tau}-\rho\right),
\end{gathered}
$$

where $\tau=\omega t$ and $\xi=k z$ are respectively the dimensionless time and distance, $\Omega=(\mu / \hbar \omega) A$ is the normalized Rabi frequency, $A$ is the electric field strength, $\omega$ is the light circular frequency, $k=\omega / c$ is the wave number in vacuum, $c$ is the speed of light, $\hbar$ is the reduced Planck constant, and $\mu$ is the dipole moment of the quantum transition. The Rabi frequency is dynamically coupled to the characteristics of the two-level system - the complex amplitude of microscopic (atomic) polarization $\rho$ and the difference between populations of the ground and excited states $w$. The efficiency of the lightmatter coupling is given by the dimensionless parameter $\alpha=$ $\omega_{L} / \omega=4 \pi \mu^{2} C / 3 \hbar \omega$, where $\omega_{L}$ is the Lorentz frequency and $C$ is the concentration (density) of active (two-level) atoms. In general, the light frequency $\omega$ is detuned from the frequency $\omega_{0}$ of the atomic resonance as described by $\delta=\left(\omega_{0}-\omega\right) / \omega$. The normalized relaxation rates of population $\gamma_{1}=1 /\left(\omega T_{1}\right)$ and polarization $\gamma_{2}=1 /\left(\omega T_{2}\right)$ are expressed by means of the longitudinal $T_{1}$ and transverse $T_{2}$ relaxation times. The influence of the polarization of the background dielectric having real-valued refractive index $n_{d}$ on the embedded active particles is taken into account by the local-field enhancement factor $l=\left(n_{d}^{2}+2\right) / 3[40,41]$.

The equilibrium population difference $w_{e q}$ will allow us to describe both gain and loss materials with the same MaxwellBloch equations (1)-(3). When the external pump is absent, the two-level atoms are in the ground state $\left(w_{e q}=1\right)$ and the medium is lossy. In the case of gain, the equilibrium population difference can be referred to as a pumping parameter. In the fully inverted medium, all atoms are excited by the pump $\left(w_{e q}=-1\right)$. For the saturated medium with both levels populated equally in the equilibrium, there are no transitions between the levels $\left(w_{e q}=0\right)$.

In the steady-state approximation, when the amplitudes of population difference, polarization, and field are time independent, one can use the effective permittivity of a two-level medium [21]

$$
\varepsilon_{e f f}=n_{d}^{2}+4 \pi \mu C \rho_{s t} / E=n_{d}^{2}+\frac{K\left(-\delta+i \gamma_{2}\right)}{1+|\Omega|^{2} / \Omega_{\mathrm{sat}}^{2}},
$$

where $\Omega_{\text {sat }}=\sqrt{\gamma_{1}\left(\gamma_{2}^{2}+\delta^{2}\right) / 4 l^{2} \gamma_{2}}$ sets the level of saturation intensity and $K=3 \omega_{L} l^{2} w_{e q} /\left[\omega\left(\gamma_{2}^{2}+\delta^{2}\right)\right]$. At the 


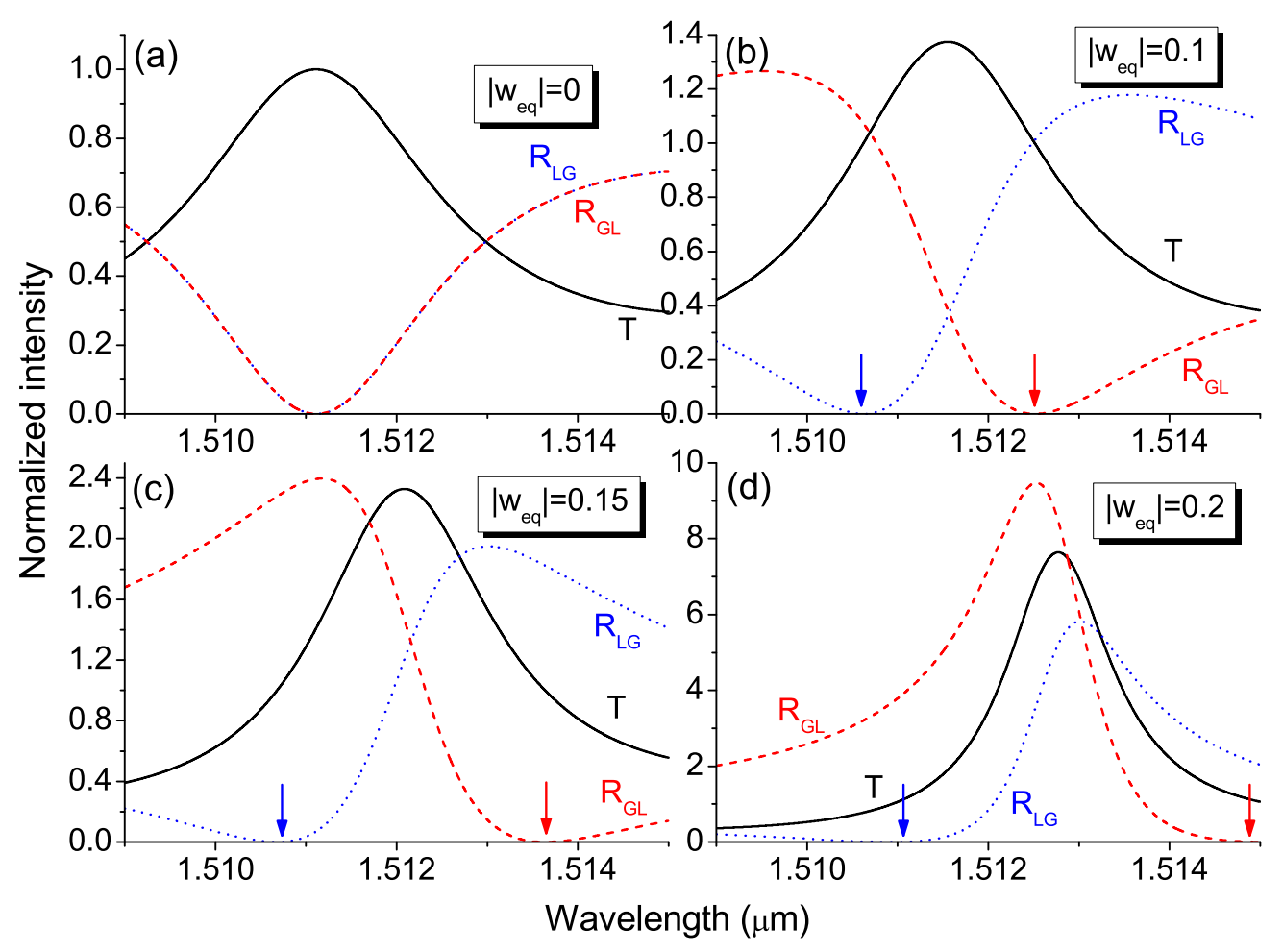

FIG. 2. Reflection (R) and transmission (T) spectra for different levels of pumping: (a) $\left|w_{e q}\right|=0$, (b) $\left|w_{e q}\right|=0.1$, (c) $\left|w_{e q}\right|=0.15$, and (d) $\left|w_{\text {eq }}\right|=0.2$. The parameters of the $\mathcal{P} \mathcal{T}$-symmetric structure are given in the text. Arrows mark anisotropic transmission resonances.

exact resonance $\delta=0$ and in approximation of low-intensity external radiation $|\Omega| \ll \Omega_{\text {sat }}$, Eq. (4) transforms to $\varepsilon_{\text {eff }} \approx$ $n_{d}^{2}+3 i l^{2} \omega_{L} T_{2} w_{e q}$. From this equation it is clear that gain and loss correspond to negative and positive $w_{e q}$, respectively. In the stationary approximation, it is straightforward to obtain a $\mathcal{P} \mathcal{T}$-symmetric structure composed of alternating layers with balanced loss $\left(\varepsilon_{e f f+}\right)$ and gain $\left(\varepsilon_{e f f-}\right)$, where

$$
\varepsilon_{e f f \pm} \approx n_{d}^{2} \pm 3 i l^{2} \omega_{L} T_{2}\left|w_{e q}\right|
$$

$\mathcal{P} \mathcal{T}$ symmetry holds true, because the necessary condition $\varepsilon(z)=\varepsilon^{*}(-z)$ is fulfilled, providing an even (odd) function of $z$ for the real (imaginary) part of the permittivity. In the Supplemental Material [42], $\mathcal{P} \mathcal{T}$ symmetry conditions are derived straight from the Maxwell-Bloch equations. It is shown that the system is $\mathcal{P} \mathcal{T}$ symmetric only in a steady state established after some transient period.

The identity of the absolute values of the imaginary parts of permittivities $\varepsilon_{e f f+}$ and $\varepsilon_{e f f-}$ can be achieved in different ways. From a practical point of view, it would be convenient to take unexcited absorbing layers $\left(w_{e q, L}=1\right)$ and pump only the amplifying layers to the level $w_{e q, G}=-\alpha_{L} / \alpha_{G}$, where $\alpha_{L}$ and $\alpha_{G}$ are the light-matter coupling coefficients for the loss and gain layers, respectively. Tuning of these coefficients can be properly carried out by affecting the concentration of active particles in both types of layers. Without imposing any restrictions, it is fair to claim within this theoretical investigation that the loss and gain layers have equal concentrations $C$ (hence, equal couplings $\alpha$ ) and absolute values of the pumping parameter $\left|w_{e q}\right|$. Some additional data on the variant with completely unexcited absorbing layers are given in Supplemental Material [42].
Equations (1)-(3) are solved numerically using the finitedifference time-domain (FDTD) approach developed in our previous publication [43] and recently adapted to study lossgain structures [21]. As an initial value of the population difference, we employ the pumping parameter, i.e., $w(t=$ $0)=w_{e q}$. Comparison of the results of numerical simulations with those of the transfer-matrix method with Eq. (5) for permittivities of loss and gain layers will unveil the limitations of applicability of the latter approach.

In this paper, we use a semiconductor doped with quantum dots as an active material and assume the condition of exact resonance $\delta=0$ is valid. It can be characterized by the following parameters $[44,45]: n_{d}=3.4, \omega_{L}=$ $10^{11} \mathrm{~s}^{-1}, T_{1}=1 \mathrm{~ns}$, and $T_{2}=0.5 \mathrm{ps}$. The gain coefficient $g=4 \pi \operatorname{Im}\left(\sqrt{\varepsilon_{\text {eff- }}}\right) / \lambda \leqslant 10^{4} \mathrm{~cm}^{-1}$ is estimated according to Eq. (5) for $\lambda \sim 1.5 \mu \mathrm{m}$ and $\left|w_{e q}\right| \leqslant 0.2$ can be realized in practice [46]. The multilayer structure contains $N=20$ unit cells. Both loss and gain layers have the same thickness, $d=$ $1 \mu \mathrm{m}$. The pumping scheme similar to that realized by Wong et al. [29] can be used in our system. It is also worth noting that the choice of materials is not unique, but the multilayer parameters and light wavelength may need to be appropriately adjusted to obtain similar results with different materials.

\section{TEMPORAL DYNAMICS OF LIGHT IN $\mathcal{P} \mathcal{T}$-SYMMETRIC PHASE}

We start our study analyzing the stationary characteristics of one-dimensional $\mathcal{P} \mathcal{T}$-symmetric structures. In the stationary mode, the transfer-matrix method for the wave propagation through multilayers with permittivities (5) is exploited. Owing to reciprocity of the system, the transmission 


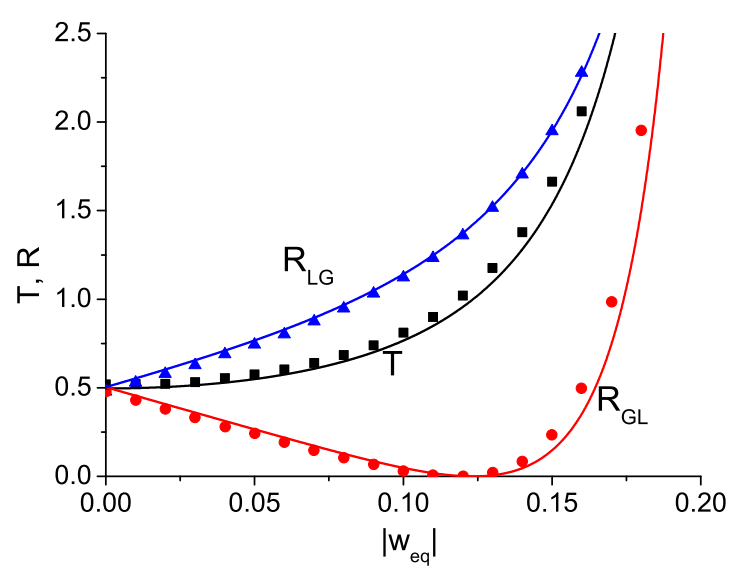

FIG. 3. Dependence of stationary levels of the reflection (R) and transmission (T) on pumping parameter $\left|w_{e q}\right|$ at the wavelength $\lambda=$ $1.513 \mu \mathrm{m}$. Symbols and lines correspond to results of numerical and transfer-matrix calculations, respectively.

of oppositely propagating (forward and backward) waves is the same, but the reflection is different. We denote these two directions of wave propagation with subscripts (loss-gain) LG and (gain-loss) GL (see Fig. 1), originating from the order of layers in the unit cell of the structure. In the case of $w_{e q}=0$ (homogeneous dielectric slab of thickness $2 \mathrm{Nd}$ ), the reflections are equal, $R_{L G}=R_{G L}$ [Fig. 2(a)]. Divergence of the curves for $R_{L G}$ and $R_{G L}$ in Figs. 2(b)-2(d) indicates existence of the $\mathcal{P} \mathcal{T}$ symmetry in full accordance with the properties of transfer matrices of such systems [10]. In these figures, one can notice another well-known feature, the so-called anisotropic transmission resonance (ATR) [10]. It emerges under the following conditions: transmission $T=1$ and one of the reflections is zero. Thus, the ATR arises for the two wavelengths marked with arrows in Fig. 2 corresponding to $R_{G L}>R_{L G}=0$ and $R_{L G}>R_{G L}=0$. Transmission exceeds unity between these points.

The increase of the pumping parameter (hence, the absolute value of the imaginary part of the slab permittivities) results in the redshift of the ATR. This means that the ATR can be reached by changing $\left|w_{e q}\right|$ at any fixed wavelength near the transmission peak. In order to examine this prediction of the transfer-matrix method, we perform numerical simulations of the Maxwell-Bloch equations (1)-(3) for a monochromatic wave $(\lambda=1.513 \mu \mathrm{m})$ propagating through the $\mathcal{P} \mathcal{T}$ symmetric multilayer with different pumping parameters. To keep the correspondence with the transfer-matrix approach, the saturation should be neglected. Here it is realized for as low an incident wave amplitude as $\Omega_{0}=10^{-5} \gamma_{2} \ll \Omega_{\text {sat }}$. The results of numerical calculations shown with symbols in Fig. 3 agree well with those of the matrix method (lines in Fig. 3). The ATR at $\left|w_{e q}\right| \sim 0.125$ is also confirmed in the FDTD calculations. Temporal dynamics shown in Fig. 4 demonstrates the transient process to the steady-state formation in the dielectric slab [in the absence of loss and gain, panel (a)] and $\mathcal{P} \mathcal{T}$-symmetric structure in conditions of the ATR [panel (b)]. Since the steady state is rapidly established, the transfer-matrix approach is well applicable in this nosaturation regime. Absence of saturation is directly demonstrated by the inset in Fig. 4, where the population difference
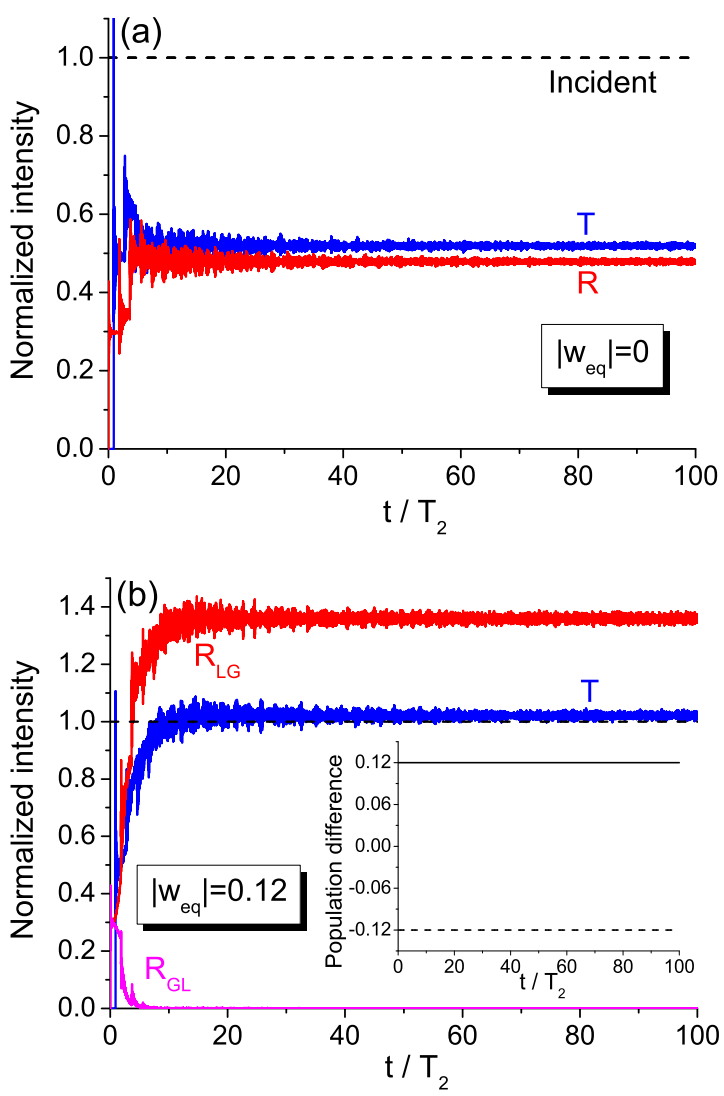

FIG. 4. Temporal dynamics of the reflected (R) and transmitted (T) intensity for the pumping parameters (a) $\left|w_{e q}\right|=0$ and (b) $\left|w_{e q}\right|=0.12$. Inset shows dynamics of population difference at the entrance of the first loss and gain layers.

does not change during establishment of the steady state. The initial transient regime is unavoidable in realistic systems. It can be studied with the FDTD method, and $\mathcal{P} \mathcal{T}$ symmetry is unreachable in this mode due to the impossibility of changing the sign of relaxation rates $\gamma_{1}$ and $\gamma_{2}$ (see Ref. [42]).

\section{PHASE TRANSITION DYNAMICS}

$\mathcal{P} \mathcal{T}$ symmetry breaking can be considered as a peculiar phase transition. It can be realized either by changing the wavelength $\lambda$ of light for a given value of pumping parameter $\left|w_{e q}\right|$, or, conversely, by changing the pumping parameter at a fixed wavelength. The latter variant is analyzed in this paper. The former one deserves a separate study, since it implies the necessity to consider the effects of frequency detuning. The criterion of the $\mathcal{P} \mathcal{T}$ symmetry breaking is usually formulated in terms of the eigenvalues $s_{1}$ and $s_{2}$ of the scattering matrix [10]. The scattering matrix connects the left and right input fields with left and right output fields. A $\mathcal{P} \mathcal{T}$-symmetric system has unimodular eigenvalues $\left|s_{1}\right|=\left|s_{2}\right|=1$ of the scattering matrix. When $\mathcal{P} \mathcal{T}$ symmetry is violated, the modules of the eigenvalues are inverse as $\left|s_{1}\right|>1$ and $\left|s_{2}\right|=1 /\left|s_{1}\right|<1$. At the points of phase transition called exceptional points, the eigenvectors of the scattering matrix coincide. Using the 

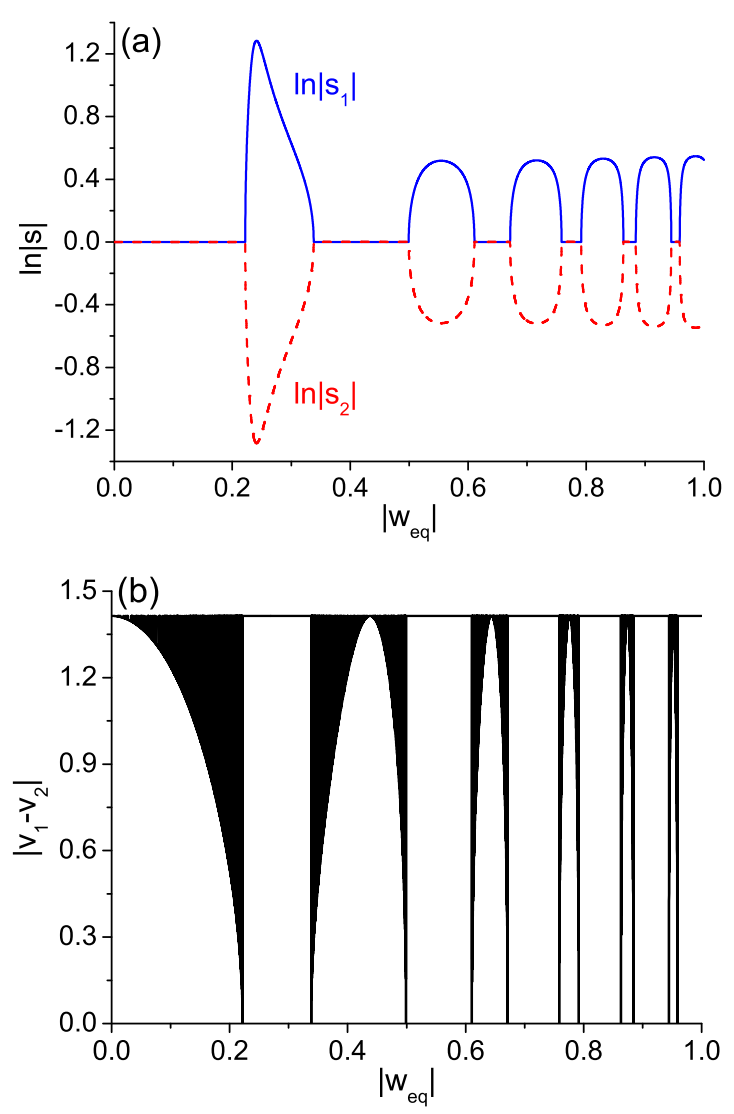

FIG. 5. (a) Logarithm of eigenvalues $s_{1}$ and $s_{2}$ and (b) difference of eigenvectors $\left|\mathbf{v}_{1}-\mathbf{v}_{2}\right|$ of the scattering matrix as a function of the pumping parameter $\left|w_{e q}\right|$.

definition of the scattering matrix [10]

$$
S=\left(\begin{array}{ll}
r_{L G} & t_{G L} \\
t_{L G} & r_{G L}
\end{array}\right),
$$

we calculate both eigenvalues and eigenvectors for the pumping parameter $\left|w_{e q}\right|$ swept through the whole interval from 0 to 1 . Here $t_{L G}, t_{G L}, r_{L G}$, and $r_{G L}$ are the transmission and reflection coefficients, which can be expressed through the respective elements of the transfer matrix $M: t_{L G}=$ $1 / M_{11}, t_{G L}=\operatorname{det}[M] / M_{11}=t_{L G}($ since $\operatorname{det}[M]=1), r_{L G}=$ $M_{21} / M_{11}$, and $r_{G L}=-M_{12} / M_{11}$. Stationary transmission and reflection are calculated as $T=\left|t_{L G}\right|^{2}=\left|t_{G L}\right|^{2}, R_{L G}=$ $\left|r_{L G}\right|^{2}$, and $R_{G L}=\left|r_{G L}\right|^{2}$. Results of transfer-matrix calculations for the multilayer structure with the permittivities (5) are shown in Fig. 5. At the first exceptional point $\left|w_{e q}\right| \approx$ 0.222 , the phase transition occurs and eigenvalues cease to be unimodular [Fig. 5(a)], whereas the difference between eigenvectors vanishes [Fig. 5(b)]. Unimodularity is violated and, therefore, $\mathcal{P} \mathcal{T}$ symmetry is broken up to the second exceptional point. The latter returns the system into the $\mathcal{P} \mathcal{T}$ symmetric state. All in all, there are a number of ranges of $\left|w_{\text {eq }}\right|$ with broken symmetry and a corresponding number of exceptional points.

The transfer-matrix approach used so far cannot describe the dynamics of the wave propagation in resonant media by its definition. Now we will employ the rigorous Maxwell-Bloch equations for studying light dynamics near an exceptional
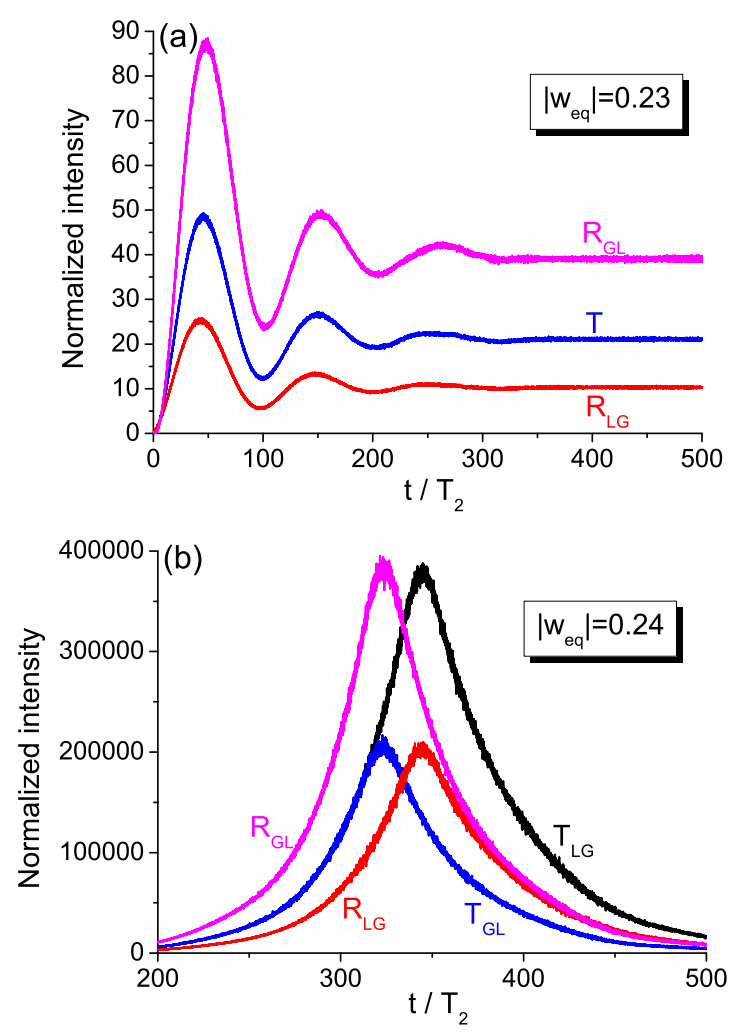

FIG. 6. Temporal dynamics of the reflected (R) and transmitted (T) intensity for pumping parameters (a) $\left|w_{e q}\right|=0.23$ and (b) $\left|w_{e q}\right|=0.24$.

point. There is a dramatic discrepancy between the two calculation techniques, when the pumping parameter approaches the exceptional point. The relative difference in transmission calculated with the help of the Maxwell-Bloch and transfermatrix approaches, $\left|T_{M B}-T_{T M}\right| / T_{M B} \approx 0.13$, results in a satisfactory agreement for $\left|w_{e q}\right|=0.22$, but its value 0.48 at $\left|w_{e q}\right|=0.23$ is unacceptably large. Such a large discrepancy stems from the qualitatively different behaviors of the system at $\left|w_{e q}\right|=0.23$ : the system is above the exceptional point according to the transfer-matrix method, whereas it is still in the $\mathcal{P} \mathcal{T}$-symmetric state according to the FDTD simulations. In fact, temporal dynamics with an established stationary state in Fig. 6(a) is distinctive for the $\mathcal{P} \mathcal{T}$ symmetry (cf. Fig. 4). Formation of the steady state after a rather short time corroborates the existence of the balance between gain and loss at $\left|w_{e q}\right|=$ 0.23 . The general tendency is that the closer to the exceptional point, the longer the transient period is. At the exceptional point [Fig. 6(b)], the field rapidly grows, changing the population difference $w$, this growth being limited by saturation.

Breaking of $\mathcal{P} \mathcal{T}$ symmetry is expected to result in the strong (exponential) amplification of propagating waves due to the fact that gain cannot be compensated with losses in this case. In Fig. 6(b), instability exhibiting very strong light amplification is observed at $\left|w_{e q}\right|=0.24$. The energy pumped in the system is promptly released as a high-intensity pulse. In concordance with Ref. [21], this regime can be called a lasinglike (or quasilasing) mode. It can be treated as a dynamical feature of the phase state of broken $\mathcal{P} \mathcal{T}$ symmetry. In contrast to the true lasing, the pulse is generated not by 


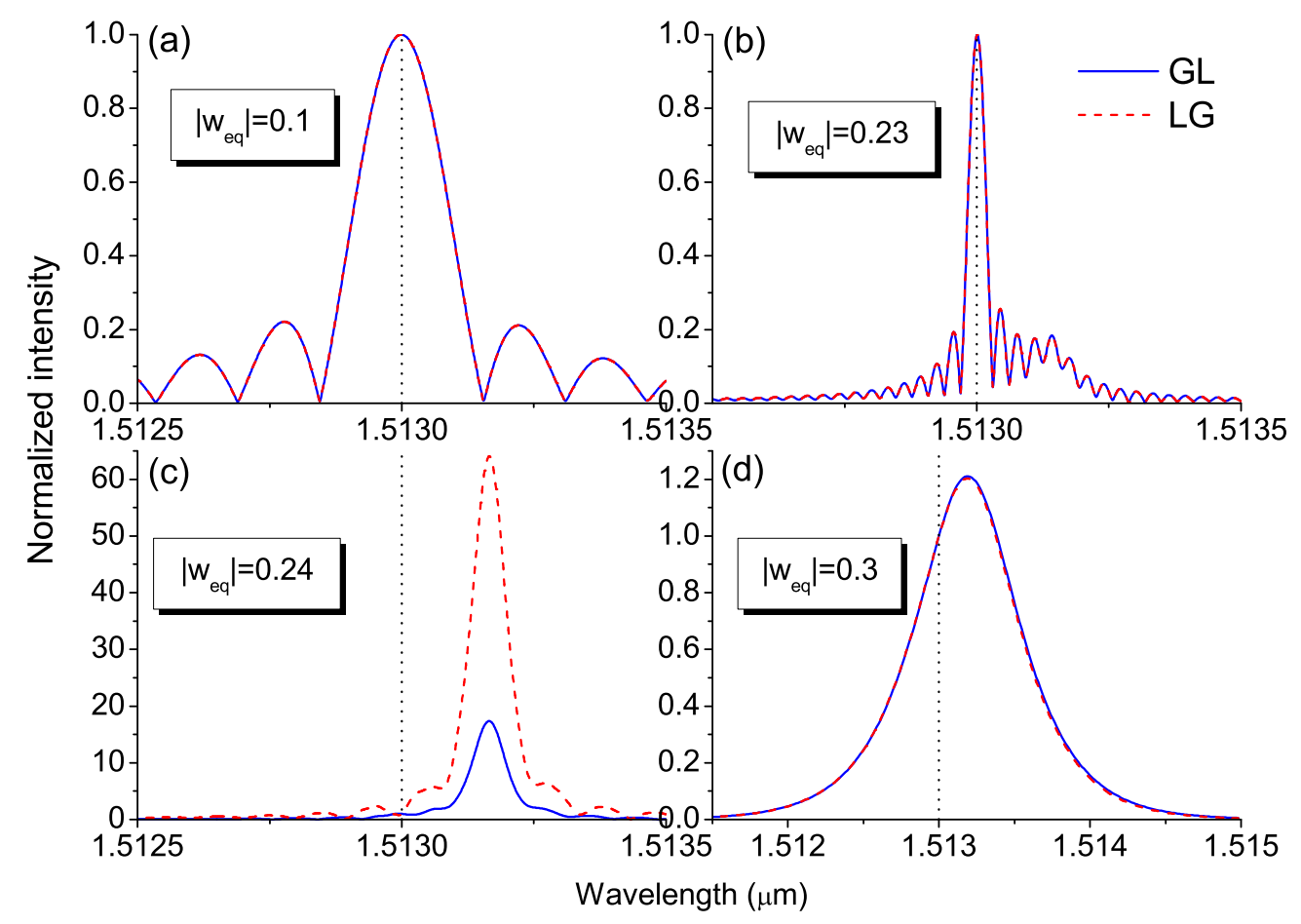

FIG. 7. Spectra of transmitted radiation for different levels of pumping: (a) $\left|w_{e q}\right|=0.1$, (b) $\left|w_{e q}\right|=0.23$, (c) $\left|w_{e q}\right|=0.24$, and (d) $\left|w_{e q}\right|=$ 0.3. The spectra are normalized on the intensity at resonant wavelength $1.513 \mu \mathrm{m}$.

small field fluctuations but rather in response to the incident wave (though the intensity of the input wave can be taken much lower, as evidenced by Supplemental Material Fig. S4 [42]). At the exceptional point, we have a very long transient period and the stationary levels of reflection and transmission are again expected to occur in the long time limit [21]. These levels strongly differ from those calculated with the transfermatrix method due to saturation development, discussed further. In other words, the resulting population difference will no longer be determined by the pumping parameter $w_{e q}$, as assumed in Eq. (5). The pulse gets shorter and more powerful with increasing $\left|w_{e q}\right|$, as evidenced by comparison of Fig. 6(b) and the Supplemental Material Fig. S1 [42]. The spectra of transmitted radiation shown in Fig. 7 indicate the shift of maximal amplification from the resonant wavelength $\lambda=1.513 \mu \mathrm{m}$ (below the exceptional point) to longer wavelengths (above the exceptional point). This redshift in the lasinglike regime can be explained by two factors: (i) higher light absorption on the resonant wavelength in the loss layers, so that amplification at the neighboring wavelengths becomes prevalent, and (ii) intensity modulation due to incomplete stationary-state establishment (see Supplemental Material Fig. S5 [42]). We should stress that although the matrix method with permittivities (5) is able to approximately determine an exceptional point, it fails in adequate description of the phase transition and in describing the temporal dynamics of light-structure interaction. In other words, the full system of Maxwell-Bloch equations should be exploited in the vicinity of the points of $\mathcal{P} \mathcal{T}$ symmetry breaking.

Strong amplification of a signal in the non- $\mathcal{P} \mathcal{T}$-symmetric phase [Fig. 6(b)] plays an important role in loss and gain saturation. Indeed, normalized light amplitude $\Omega$ inside the system is not much less than $\Omega_{\text {sat }}$ anymore. The population difference preserves its initial value $w(t)=w_{\text {eq }}$ in the $\mathcal{P} \mathcal{T}$ symmetric state below the exceptional point (see the dashed lines in Fig. 8, for $\left|w_{e q}\right|=0.23$ ). However, fluctuations of the population difference due to saturation occur above the exceptional point, at $\left|w_{e q}\right|=0.24$, in both LG (in the loss layer) and GL (in the gain layer) configurations. Saturation imposes a constraint on further increase of light intensity, as evidenced by the coincidence of the intensity peak in Fig. 6(b) and saturation development in time in Fig. 8. The fluctuations indicate that the $\mathcal{P} \mathcal{T}$ symmetry is broken through violation of the necessary condition $\varepsilon(z)=\varepsilon^{*}(-z)$, and loss and gain are

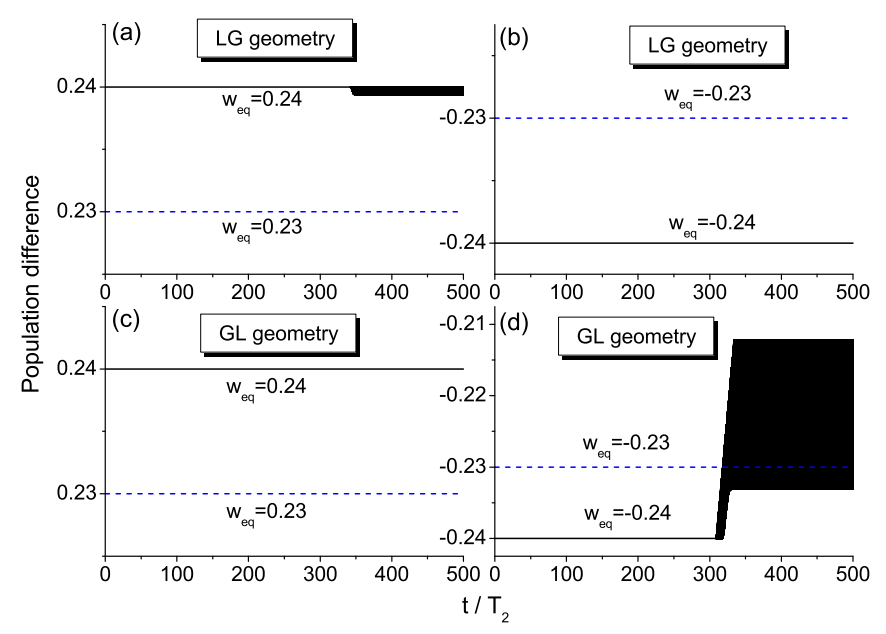

FIG. 8. Temporal dynamics of the population difference in the first unit cell of LG and GL structures at pumping parameters $\left|w_{\text {eq }}\right|=$ 0.23 and 0.24: $(\mathrm{a}, \mathrm{c}) w(t)$ in the loss layer and $(\mathrm{b}, \mathrm{d}) w(t)$ in the gain layer. 
not balanced anymore. Saturation also leads to the irreversible phase transition in the system: the return of the system to the $\mathcal{P} \mathcal{T}$-symmetric state predicted by the stationary theory at larger pumping parameters (see Fig. 5) is impossible, since Eq. (5) is not valid anymore. Direct FDTD calculations for $\left|w_{e q}\right|>0.24$ (see Supplemental Material Fig. S1 [42]) do support this conclusion, resulting in the lasinglike dynamics similar to those shown in Fig. 6(b).

Broken $\mathcal{P} \mathcal{T}$ symmetry drastically affects the dynamics of the transmitted and reflected waves. Owing to the nonlinear process of saturation, the transmission becomes asymmetric, $T_{L G} \neq T_{G L}$, i.e., the multilayer structure is nonreciprocal. Usually the saturation-induced nonreciprocity is introduced through the nonlinear permittivity Eq. (4) [35,36], but the solution of dynamic Eqs. (1)-(3) is more accurate and informative. In the saturation regime the system is non-Hermitian, but it can be linearized to a $\mathcal{P} \mathcal{T}$-symmetric multilayer [36].

Intensities of the pulses escaping the system do not depend on the direction of incident light: almost the same pulses are emitted from the gain and loss ends of the multilayer after reversing the input light direction $\left(T_{L G}=R_{G L}\right.$ and $\left.T_{G L}=R_{L G}\right)$ as shown in Fig. 6(b). In other words, the direction of the output pulses is locked by $\mathcal{P} \mathcal{T}$ symmetry breaking. This locking can be presumably caught only within the dynamical calculations, because it has not been reported earlier. Nonreciprocal transmission is accompanied by the propagation direction locking at higher pumping as well, which is demonstrated in Supplemental Material Fig. S1 [42] for $\left|w_{e q}\right|=0.3$ and 0.4. Locking of the light propagation directions can be viewed as a possible basis for peculiar all-optical diodes and transistors.

It should be emphasized that the saturation is not the reason for this locking. In order to demonstrate this, we consider wave propagation in the saturation regime (for initial amplitude $\Omega_{0}=10^{-2} \gamma_{2}$ ) which breaks $\mathcal{P} \mathcal{T}$ symmetry at every value of the pumping parameter. Nonreciprocity of transmission due to saturation is clearly seen in Fig. 9, but the direction locking of output pulses is missing. Therefore, the $\mathcal{P} \mathcal{T}$ symmetry breaking is necessary for this locking to occur.

Behavior similar to that described above generally occurs near the exceptional points, as evidenced by Figs. S2, S3, and S4 in the Supplemental Material [42]. In particular, the number of layers controls position of the exceptional point: when the structure length is decreased, higher pumping is needed for $\mathcal{P} \mathcal{T}$ symmetry breaking (Fig. S2 of the Supplemental Material [42]) and vice versa. Although the system's response is generally very complex due to interplay of the loss/gain and multilayer resonances [37], results similar to those discussed above are expected for other parameters of the structure and proper operating frequency. Such scalability is encouraging for designing realistic realizations of $\mathcal{P} \mathcal{T}$-symmetric multilayers. We would like to emphasize that another model of $\mathcal{P} \mathcal{T}$-symmetric multilayers with unexcited absorbing layers mentioned in Sec. II provides similar results (see Supplemental Material Fig. S6 [42]).

\section{CONCLUSION}

We have analyzed temporal dynamics of light in $\mathcal{P} \mathcal{T}$ symmetric periodic multilayers, the gain and loss slabs being modeled as a resonant medium. Light-matter interactions
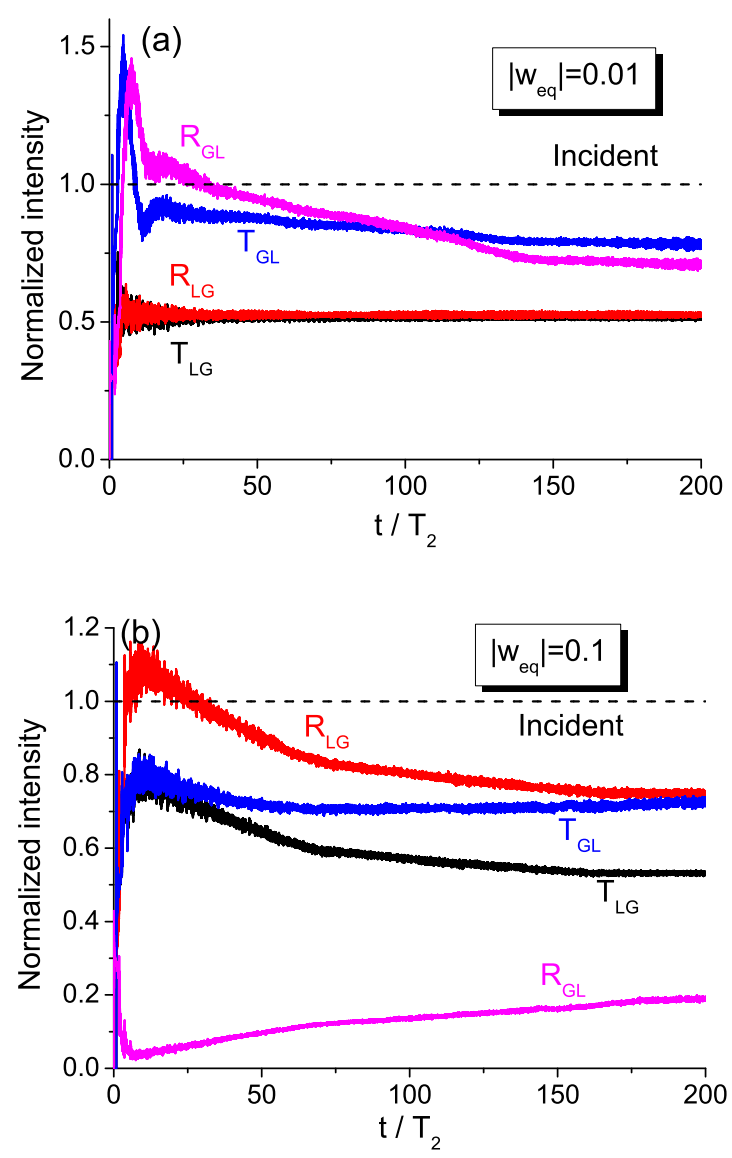

FIG. 9. Temporal dynamics of the reflected (R) and transmitted ( $\mathrm{T}$ ) intensity for the pumping parameter (a) $\left|w_{\text {eq }}\right|=0.01$ and (b) $\left|w_{\text {eq }}\right|=0.1$ in the case of the wave with relatively large initial amplitude $\Omega_{0}=10^{-2} \gamma_{2}$.

in the resonant media are described by the Maxwell-Bloch equations, which are simulated numerically to provide deeper insight into transition dynamics between the $\mathcal{P} \mathcal{T}$-symmetric and $\mathcal{P} \mathcal{T}$-broken phases. In particular, predictions of the stationary transfer-matrix method are shown to be inadequate in the vicinity of the exceptional points. We feature the socalled lasinglike regime in the $\mathcal{P} \mathcal{T}$ symmetry broken state characterized by emission of powerful pulses of radiation and development of saturation. The latter is the reason for phase transition irreversibility-that is, the system cannot return to the $\mathcal{P} \mathcal{T}$-symmetric state for the pumping parameters above the exceptional point. In the $\mathcal{P} \mathcal{T}$-broken phase, the direction of pulses escaping the system is found to be locked by the $\mathcal{P} \mathcal{T}$ symmetry breaking, meaning that the intensities of two output waves are independent of the direction of the incident radiation. The approach based on the Maxwell-Bloch equations seems to be rather general and applicable to structures with other geometries, e.g., coupled ring resonators [26]. We envisage its application to investigation of other effects near the exceptional points, such as coherent perfect absorption (antilasing) [29-31]. Intricate interplay between loss and gain in $\mathcal{P} \mathcal{T}$-symmetric systems opens up new opportunities for constructing photonic devices for optical communications, computing, and sensing. The approach proposed here is expected to be useful to realize some of these diverse functionalities. 


\section{ACKNOWLEDGMENTS}

The work was supported by the Belarusian Republican Foundation for Fundamental Research (Projects No. F16K016 and No. F18R-021), the Russian Foundation for Basic Research (Projects No. 18-02-00414, No. 18-52-00005, and No. 18-32-00160), Ministry of Education and Science of the Russian Federation (GOSZADANIE, Grant No. 3.4982.2017/6.7), Government of Russian Federation (Grant
No. 08-08), and the Israeli Ministry of Trade and Labor Kamin Program (Grant No. 62045). Numerical simulations of light interaction with resonant media were supported by the Russian Science Foundation (Project No. 17-72-10098). The calculations of field distributions were supported by the Russian Science Foundation (Project No. 16-12-10287). Partial financial support from Villum Fonden (the DarkSILD project) is acknowledged.
[1] A. A. Zyablovsky, A. P. Vinogradov, A. A. Pukhov, A. V. Dorofeenko, and A. A. Lisyansky, Phys. Usp. 57, 1063 (2014).

[2] L. Feng, R. El-Ganainy, and L. Ge, Nat. Photon. 11, 752 (2017).

[3] R. El-Ganainy, K. G. Makris, M. Khajavikhan, Z. H. Musslimani, S. Rotter, and D. N. Christodoulides, Nat. Phys. 14, 11 (2018).

[4] C. M. Bender and S. Boettcher, Phys. Rev. Lett. 80, 5243 (1998)

[5] C. M. Bender, Rep. Prog. Phys. 70, 947 (2007).

[6] R. El-Ganainy, K. G. Makris, D. N. Christodoulides, and Z. H. Musslimani, Opt. Lett. 32, 2632 (2007).

[7] K. G. Makris, R. El-Ganainy, D. N. Christodoulides, and Z. H. Musslimani, Phys. Rev. Lett. 100, 103904 (2008).

[8] C. E. Rüter, K. G. Makris, R. El-Ganainy, D. N. Christodoulides, M. Segev, and D. Kip, Nat. Phys. 6, 192 (2010).

[9] F. Yang and Z. L. Mei, Sci. Rep. 5, 14981 (2015).

[10] L. Ge, Y. D. Chong, and A. D. Stone, Phys. Rev. A 85, 023802 (2012).

[11] Z. Lin, H. Ramezani, T. Eichelkraut, T. Kottos, H. Cao, and D. N. Christodoulides, Phys. Rev. Lett. 106, 213901 (2011).

[12] R. Fleury, D. L. Sounas, and A. Alù, Phys. Rev. Lett. 113, 023903 (2014).

[13] Ya. V. Kartashov, C. Hang, V. V. Konotop, V. A. Vysloukh, G. Huang, and L. Torner, Laser Photon. Rev. 10, 100 (2016).

[14] S. V. Suchkov, A. A. Sukhorukov, J. Huang, S. V. Dmitriev, C. Lee, and Yu. S. Kivshar, Laser Photon. Rev. 10, 177 (2016).

[15] V. V. Konotop, J. Yang, and D. A. Zezyulin, Rev. Mod. Phys. 88, 035002 (2016).

[16] A. T. Lupu, H. Benisty, and A. V. Lavrinenko, IEEE J. Sel. Topics Quantum Electron. 22, 35 (2016).

[17] C. Hang and G. Huang, Adv. Phys. X 2, 737 (2017).

[18] S. Weimann, M. Kremer, Y. Plotnik, Y. Lumer, S. Nolte, K. G. Makris, M. Segev, M. C. Rechtsman, and A. Szameit, Nat. Mater. 16, 433 (2017).

[19] Y. Shen, X. H. Deng, and L. Chen, Opt. Express 22, 19440 (2014).

[20] J. Ramirez-Hernandez, F. M. Izrailev, and N. M. Makarov, Phys. Rev. A 96, 013856 (2017).

[21] D. V. Novitsky, V. R. Tuz, S. L. Prosvirnin, A. V. Lavrinenko, and A. V. Novitsky, Phys. Rev. B 96, 235129 (2017).

[22] M. Lawrence, N. Xu, X. Zhang, L. Cong, J. Han, W. Zhang, and S. Zhang, Phys. Rev. Lett. 113, 093901 (2014).

[23] A. U. Hassan, B. Zhen, M. Soljacic, M. Khajavikhan, and D. N. Christodoulides, Phys. Rev. Lett. 118, 093002 (2017).
[24] W. Chen, S. K. Özdemir, G. Zhao, J. Wiersig, and L. Yang, Nature (London) 548, 192 (2017).

[25] H. Hodaei, A. U. Hassan, S. Wittek, H. Garcia-Gracia, R. El-Ganainy, D. N. Christodoulides, and M. Khajavikhan, Nature (London) 548, 187 (2017).

[26] L. Feng, Z. J. Wong, R.-M. Ma, Y. Wang, and X. Zhang, Science 346, 972 (2014).

[27] H. Hodaei, M.-A. Miri, M. Heinrich, D. N. Christodoulides, and M. Khajavikhan, Science 346, 975 (2014).

[28] Z. Gu, N. Zhang, Q. Lyu, M. Li, S. Xiao, and Q. Song, Laser Photonics Rev. 10, 588 (2016).

[29] Z. J. Wong, Y.-L. Xu, J. Kim, K. O’Brien, Y. Wang, L. Feng, and X. Zhang, Nat. Photonics 10, 796 (2016).

[30] Y. D. Chong, L. Ge, H. Cao, and A. D. Stone, Phys. Rev. Lett. 105, 053901 (2010).

[31] S. Longhi, Phys. Rev. A 82, 031801(R) (2010).

[32] T. Goldzak, A. A. Mailybaev, and N. Moiseyev, Phys. Rev. Lett. 120, 013901 (2018).

[33] H. Ramezani, T. Kottos, R. El-Ganainy, and D. N Christodoulides, Phys. Rev. A 82, 043803 (2010).

[34] S. Phang, A. Vukovic, H. Susanto, T. M. Benson, and P. Sewell, Opt. Lett. 39, 2603 (2014).

[35] X. Liu, S. Dutta Gupta, and G. S. Agarwal, Phys. Rev. A 89, 013824 (2014).

[36] D. R. Barton III, H. Alaeian, M. Lawrence, and J. Dionne, Phys. Rev. B 97, 045432 (2018).

[37] P. Witoński, A. Mossakowska-Wyszyńska, and P. Szczepański, IEEE J. Quantum Electron. 53, 2100111 (2017).

[38] P. Lodahl, S. Mahmoodian, S. Stobbe, A. Rauschenbeutel, P. Schneeweiss, J. Volz, H. Pichler, and P. Zoller, Nature (London) 541, 473 (2017).

[39] D. V. Novitsky, Phys. Rev. A 84, 013817 (2011).

[40] M. E. Crenshaw, Phys. Rev. A 78, 053827 (2008).

[41] N. Bloembergen, Nonlinear Optics (Benjamin, New York, 1965).

[42] See Supplemental Material at http://link.aps.org/supplemental/ 10.1103/PhysRevB.98.125102 for derivation of the conditions for $\mathcal{P} \mathcal{T}$ symmetry, additional calculations of temporal dynamics of light in $\mathcal{P} \mathcal{T}$ symmetry broken phases, and calculations for the case of completely unexcited absorbing layers.

[43] D. V. Novitsky, Phys. Rev. A 79, 023828 (2009).

[44] Handbook of Optical Constants of Solids, edited by E. D. Palik (Academic Press, San Diego, CA, 1998).

[45] J.-C. Diels and W. Rudolph, Ultrashort Laser Pulse Phenomena, 2nd ed. (Academic Press, San Diego, CA, 2006).

[46] V. E. Babicheva, I. V. Kulkova, R. Malureanu, K. Yvind, and A. V. Lavrinenko, Photonics Nanostruct. Fundam. Appl. 10, 389 (2012). 